EPJ Web of Conferences 29, 00027 (2012)

DOI: $10.1051 /$ epjconf/20122900027

(C) Owned by the authors, published by EDP Sciences, 2012

\title{
Contribution to the study of electronic structure of crystalline semiconductors (Si, Ge, GaAs, Gap , ZnTe, ZnSe)
}

\author{
N. Mahi , , and B. Bouhafs \\ Université Abou Bekr Belkaid-Tlemcen, Faculté des Sciences, Laboratoire de physique théorique B.P.230 \\ Tlemcen, 13000 (Alegria)
}

\begin{abstract}
The band structure of semiconductors was described by several theorists since the Fifties. The main objective of the present paper is to do a comparative study between various families of semi-conductors IV ( $\mathrm{Si}, \mathrm{Ge})$, III-V (GaAs, GaP) and II-VI (ZnSe, ZnTe) with both methods; tight Binding ${ }^{1}$ method and pseudo potential method ${ }^{2}$. This work enables us to understand as well as the mechanism of conduction process in these semiconductors and powers and limits of the above methods. The obtained results allow to conclude that both methods are in a good agreement to describe the morphology of band structures of the cited semiconductors. This encourages us to study in the future the electronic behaviour through the structure of bands for more complex systems such as the heterostructures.
\end{abstract}

\section{The Tight Binding method}

To find the properties of electrons which determine the nature of many effects observed experimentally, it is necessary to know the band structure (indexed by n) of the solid or the energetic dependency $E_{n}(\vec{k})$ versus the electron wave vector, $\vec{k}$. In the alone electron approximation (AEA), the energy spectrum of an electron in a crystal can be determined from the Schrödinger equation:

$$
\left[-\frac{\hbar^{2}}{2 m} \nabla^{2}+U(\vec{r})\right] \psi(\vec{r})=E \psi(\vec{r})
$$

where $U(\vec{r})$ is a periodic potential exerted on the electron in the crystal. We can accept the idea that the solution of equation (1) can be expanded in a series of a complete set of functions. The latter will be the desired solution as:

$$
\psi(\vec{r})=\sum_{i=1}^{\infty} c_{i} \varphi_{i}(\vec{r})
$$

If we limit ourselves to a finite number of terms in series, one can have an approximate solution of the problem. In practice, one selects a certain set not necessarily full of function $\varphi_{1}, \varphi_{2}, \ldots . \varphi_{i}$ appropriate to solve the problem with boundary conditions. So, we construct a linear combination of these functions as a form:

$$
\psi(\vec{r})=\sum_{i=1}^{l} c_{i} \varphi_{i}(\vec{r})
$$

\footnotetext{
1 e-mail : mahidoc2@gmail.com

Article available at http://www.epj-conferences.org or http://dx.dol.org/10.1051/epjcont/20122900027
} 
The efforts of researchers in the field of methods for calculating electronic spectra have been directed to find a procedure that constructs background functions $\varphi_{\mathrm{i}}$. In addition, functions $\varphi_{i}$ should be chosen, so that calculations of the electronic wave functions $\psi$ are very simples. The nature of test functions used for computation can serve as a characteristic that distinguishes one method from another.

Linear combination of atomic orbital (LCAO) method or tight-binding has been proposed for the first time by Bloch [1]. It involves to build LCAO located on different atoms of the crystal. The corresponding coefficients represent the values of the plane wave $\exp (i \vec{k} \vec{R})$ at different positions $\vec{R}$ on which the atoms are located. LCAO method can be used as an interpolation method. This means that one can easily gets solutions of energy bands in an arbitrary point in the Brillouin's zone, whereas in most other approximation methods, it remains difficult, except at certain symmetry points of the Brillouin's zone.

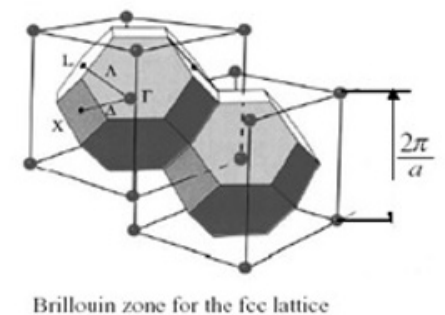

Brillouin zone for the fee lattice
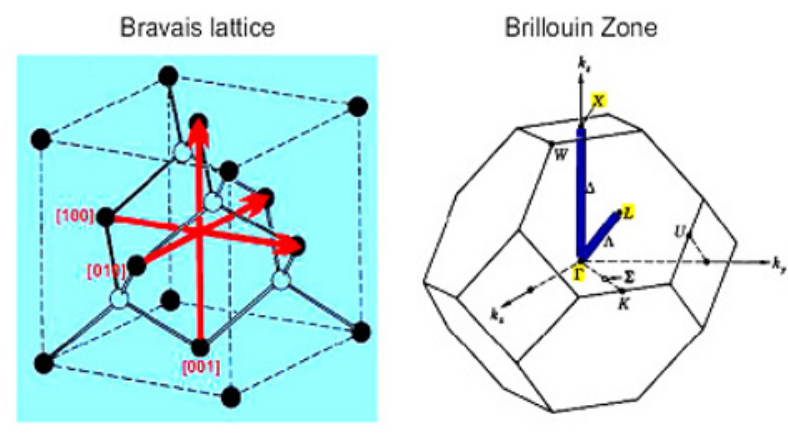

Figure 1. Brillouin's zone of a face-centered cubic lattice lines connecting points of high symmetry defines the base's domain.

Point $\Gamma$ is the center of the Brillouin's zone.

Point $\mathrm{X}$ is zone boundary in the direction $\langle 100\rangle$ and equivalent directions.

Point $\mathrm{L}$ is the zone boundary in the direction $\langle 111\rangle$ and equivalent directions

Point $\mathrm{K}$ is the zone boundary in the direction $\langle 110\rangle$ and equivalent directions.

\section{Models used in the Tight Binding method}

\subsection{Model of orbitals related B.O.M (Harrison 1973)}

The energy bands represent the electronic structure of solids. For bands obtained by the LCAO have two fundamental obstacles to understand the binding properties in solids [2]. First, the binding properties are related to the total energy and therefore the calculation of the integral of energy according to the occupied bands. This can be completed digitally in the following description as LCAO analytical forms of energy can exist only along certain symmetry directions. The second is more fundamental: the energy bands can be obtained only for perfect crystals, while several interesting binding properties are related to surfaces, defect, deformed networks, or impurities, making calculations very complicated. To overcome this, we use the approximation related orbitals (BOM). In the context of the Hamiltonian matrix according to Harrison [3], the orbital approximation is related to neglect all the matrix elements representing the coupling between the binding states and anti-bonding states. This reduces the Hamiltonian matrix to a matrix of the valence band and a matrix of the conduction band that are not coupled together, which in principle are independently diagonalized. 
In zinc blend structure, the unit cell contains two types of atoms, an anion and a cation. The first stage of the model is the construction of hybrid type $\mathrm{sp}^{3}$ on each atom oriented toward the four nearest neighbors [4].

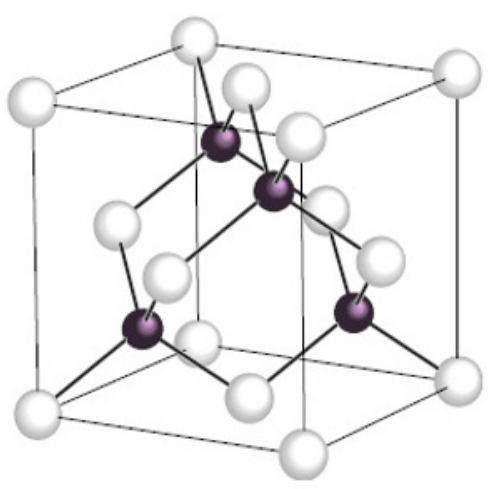

Figure2. Zinc Blende structure for semiconductor type III.-V and II-VI it is composed of two sub-networks centered cubic faces offset a quarter of the main diagonal of the cube, and one occupied by the anion (As, $\mathrm{P}, \mathrm{Te}$ and $\mathrm{Se}$ ) and the other by the cation $(\mathrm{Ga}, \mathrm{Zn})$

\subsection{Model of Vogl (1983)}

In this model, we maintain the building nearest neighbors by the model (BOM). In this case, the chemical divisions are preserved and are revealed when we want to successfully reproduce the low conduction bands which are unoccupied. As it was the case for the occupied valence bands.

The main contribution of this model is the introduction of a state (s) excited ( $\left.\mathrm{s}^{*}\right)$ on each atom. In fact, for silicon, for example, the excited state $\left(s^{*}\right)$ will be coupled with the conduction states anti-bonding type $(p)$ and near the point $\mathrm{X}$ and $\mathrm{L}$ of the Brillouin's zone. This thus requires these states to lower energy and indirect gaps will be reproduced at these points. Therefore, we built this way the new database $\mathrm{sp}^{3} \mathrm{~s}^{*}$ that will be suitable for all semiconductors and materials with the zinc blend or diamond structure.

The addition of the state $\left(\mathrm{s}^{*}\right)$ increase the base with simple orbital (d) which would make it necessary to treat the angular momentum states higher

Table .1 The Hamiltonian matrix $s p^{3} s^{*}$ (10X 10) zinc blend structure by the approximation of Tight Binding.

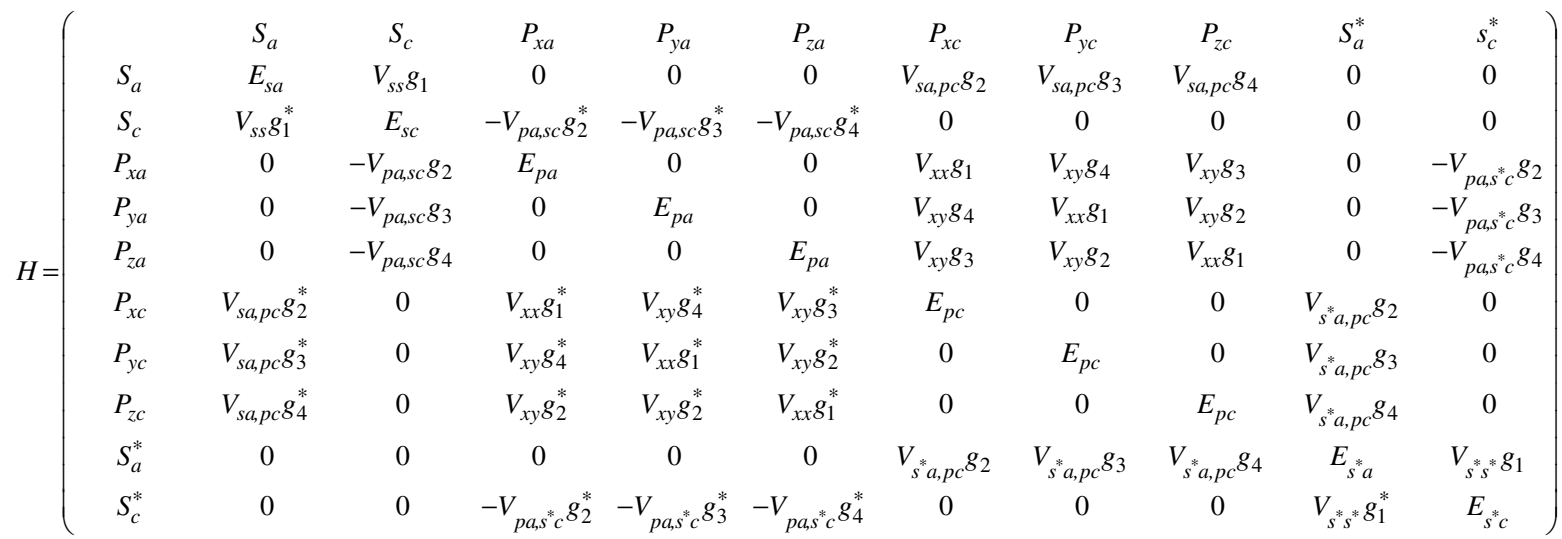

Thus, we obtain the band's structure of a semiconductor zinc blend structure by diagonalizing the above matrix. 
Table .2 The empirical parameters of the Hamiltonian matrix in unit of eV.

\begin{tabular}{|c|c|c|c|c|c|c|}
\hline & $\mathbf{S i}$ & Ge & GaAs & GaP & $\begin{array}{c}\text { ZnTe } \\
(a)\end{array}$ & ZnSe \\
\hline$E_{s a}$ & -4.2000 & -5.8800 & -8.3431 & -8.1124 & -9.9332 & -11.8383 \\
\hline$E_{p a}$ & 1.7150 & 1.6100 & 1.0414 & 1.1250 & 1.5441 & 1.5072 \\
\hline$E_{s c}$ & -4.2000 & -5.8800 & -2.6569 & -2.1976 & -0.3889 & 0.0183 \\
\hline$E_{p c}$ & 1.7150 & 1.6100 & 3.6686 & 4.1150 & 4.1508 & 5.9928 \\
\hline$E_{s^{*} a}$ & 6.6850 & 6.3900 & 8.5914 & 8.5150 & 6.9861 & 7.5872 \\
\hline$E_{s}{ }^{*} c$ & 6.6850 & 6.3900 & 6.7386 & 7.1850 & 8.0949 & 8.9928 \\
\hline$V_{s S}$ & -8.3000 & -6.7800 & -6.4513 & -7.4709 & -4.7948 & -6.2163 \\
\hline$V_{x x}$ & 1.7150 & 1.6100 & 1.9546 & 2.1516 & 2.3357 & 3.0054 \\
\hline$V_{x y}$ & 4.5750 & 4.9000 & 5.0779 & 5.1369 & 3.7916 & 5.9942 \\
\hline$V_{s a, p c}$ & 5.7292 & 5.4649 & 4.4800 & 4.2771 & 0.8580 & 3.4980 \\
\hline$V_{s c, p a}$ & 5.7292 & 5.4649 & 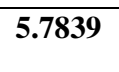 & 6.3190 & -5.0530 & 6.3191 \\
\hline$V_{s}{ }^{*} a, p c$ & 5.3749 & 5.2191 & 4.8422 & 4.6541 & 2.0970 & 2.5891 \\
\hline$V_{p a, s^{*} c}$ & 5.3749 & 5.2191 & 4.8077 & 5.0950 & -2.4723 & 3.9533 \\
\hline
\end{tabular}

(a):[5]

\subsection{Pseudo potential theory}

The pseudo potential theory shows that coefficients of the series of plane wave function orthogonalized plane waves (OPW) used to ensure an orthogonality of eigen-states can be grouped together to create a repulsive potential due to interactions of valence's electrons. The sum of this potential with the attractive potential of electrons of the heart creates a potential very low and even negligible called pseudo potential. This potential can be treated using the theory of free electrons or other techniques to solve the Schrödinger equation [6]. We write:

$$
\left(-\frac{\hbar^{2}}{2 m} \Delta+V_{c}+V_{R}\right) \Phi=\left(-\frac{\hbar^{2}}{2 m} \Delta+V^{P}\right) \Phi=E \Phi
$$

where $\mathrm{V}_{\mathrm{C}}$ and $\mathrm{V}_{\mathrm{R}}$ are the attractive and repulsive potentials respectively and $\mathrm{V}^{\mathrm{P}}$ being the pseudo potential which becomes weakly attractive in the region of the heart and weakly repulsive in the region of valence's electrons. 


\section{The pseudo-potential form factors of semiconductor diamond and Zinc Blend}

The main reason of the pseudo potential tools is that the limited number of these form factors and is sufficient to calculate the band structure. In semiconductors of diamond structure Si and Ge as the only three pseudo-potential form factors are sufficient. In the case of semi-conductor structure Zinc Blende numbers of pseudo-potential form factors will be doubled to six.

$$
V_{g}=\frac{1}{\Omega} \int\left[V_{a}\left(\vec{r}-\vec{r}_{a}\right)+V_{b}\left(\vec{r}-\vec{r}_{b}\right)\right] \exp [-i \vec{g} \cdot \vec{r}] d \vec{r}
$$

There are two types of atoms (a) and (b) in the unit cell, pseudo atomic potentials of these two atoms are denoted $V_{a}\left(\vec{r}-\vec{r}_{a}\right)$ and $V_{b}\left(\vec{r}-\vec{r}_{b}\right)$ oar $\vec{r}_{a}$ and $\vec{r}_{b}$ are the positions of two atoms in the unit cell. We will define the components of symmetric and antisymmetric form factors of pseudo potentials by the following relationships:

$$
\begin{aligned}
& V_{g}^{s}=\frac{1}{\Omega} \int\left(V_{a}+V_{b}\right) \exp (-i \vec{g} \cdot \vec{r}) d \vec{r} \\
& \text { and } \\
& V_{g}^{a}=\frac{1}{\Omega} \int\left(V_{a}-V_{b}\right) \exp (-i \vec{g} \cdot \vec{r}) d \vec{r} \\
& V_{g}=V_{g}^{s} \cos (\vec{g} \cdot \vec{s})-i V_{g}^{a} \sin (\vec{g} \cdot \vec{s})
\end{aligned}
$$

Table .3: The pseudo potential form factors of several semiconductors of group IV ( $\mathrm{Si}$ and Ge) and group III-V (GaAs and $\mathrm{GaP}$ ) and group II-VI (ZnTe and ZnSe) (given per unit Rydberg = 13.6 eV). [6]

\begin{tabular}{|c|c|c|c|c|c|c|}
\hline & $V_{3}^{s}$ & $V_{8}^{s}$ & $V_{11}^{s}$ & $V_{3}^{a}$ & $V_{4}^{a}$ & $V_{11}^{a}$ \\
\hline $\mathrm{Si}$ & -0.211 & 0.04 & 0.08 & 0 & 0 & 0 \\
\hline $\mathrm{Ge}$ & -0.269 & 0.038 & 0.035 & 0 & 0 & 0 \\
\hline $\mathrm{GaAs}$ & -0.252 & 0 & 0.08 & 0.068 & 0.066 & 0.012 \\
\hline $\mathrm{Gap}$ & -0.249 & 0.017 & 0.083 & 0.081 & 0.055 & 0.003 \\
\hline $\mathrm{ZnTe}$ & -0.228 & -0.012 & 0.059 & 0.109 & 0.105 & -0.012 \\
$(b)$ & & & & & & \\
\hline $\mathrm{ZnSe}$ & -0.23 & 0.01 & 0.06 & 0.18 & 0.12 & 0.03 \\
\hline
\end{tabular}

(b): [7] 


\section{Results and discussion}
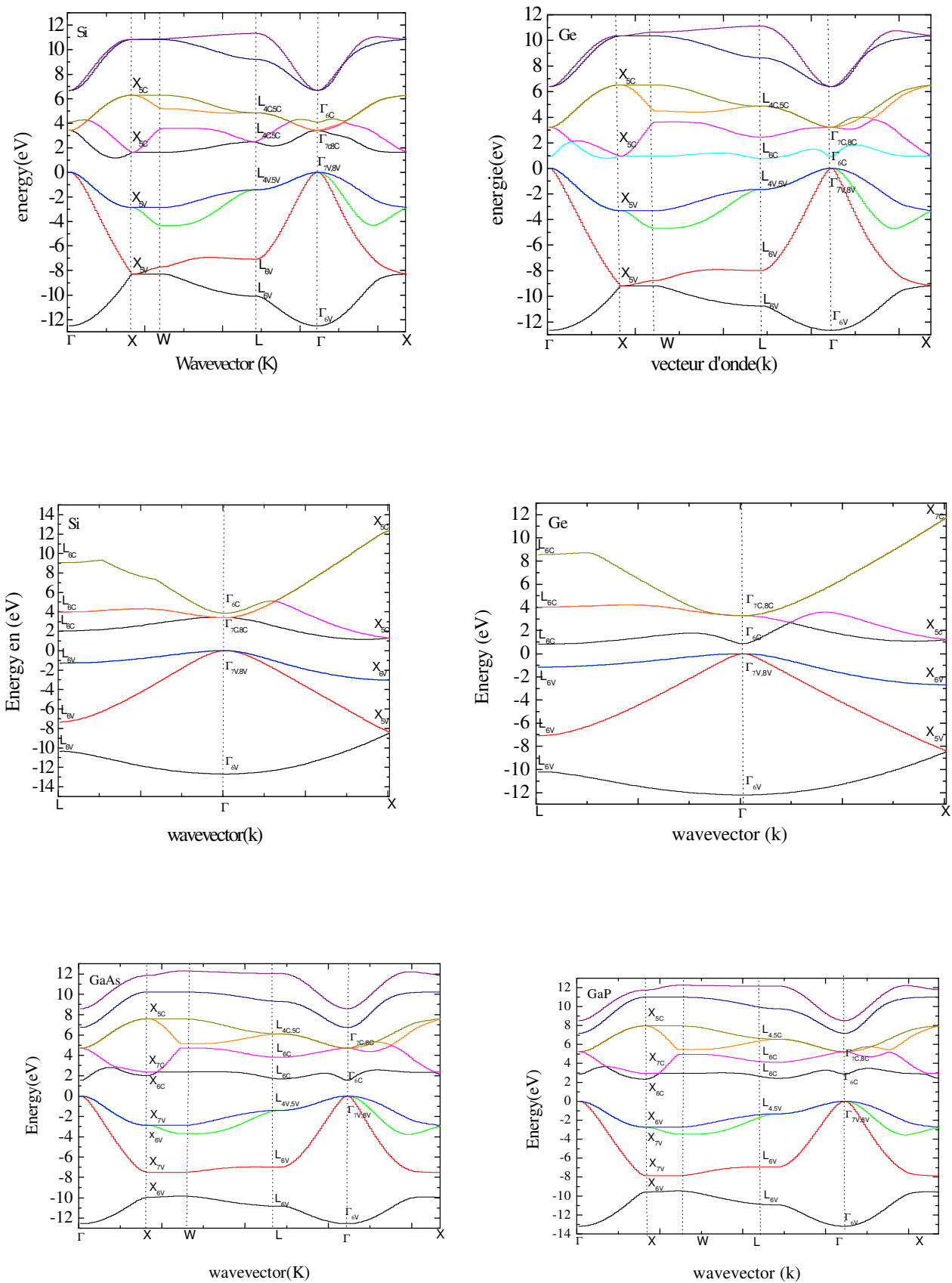

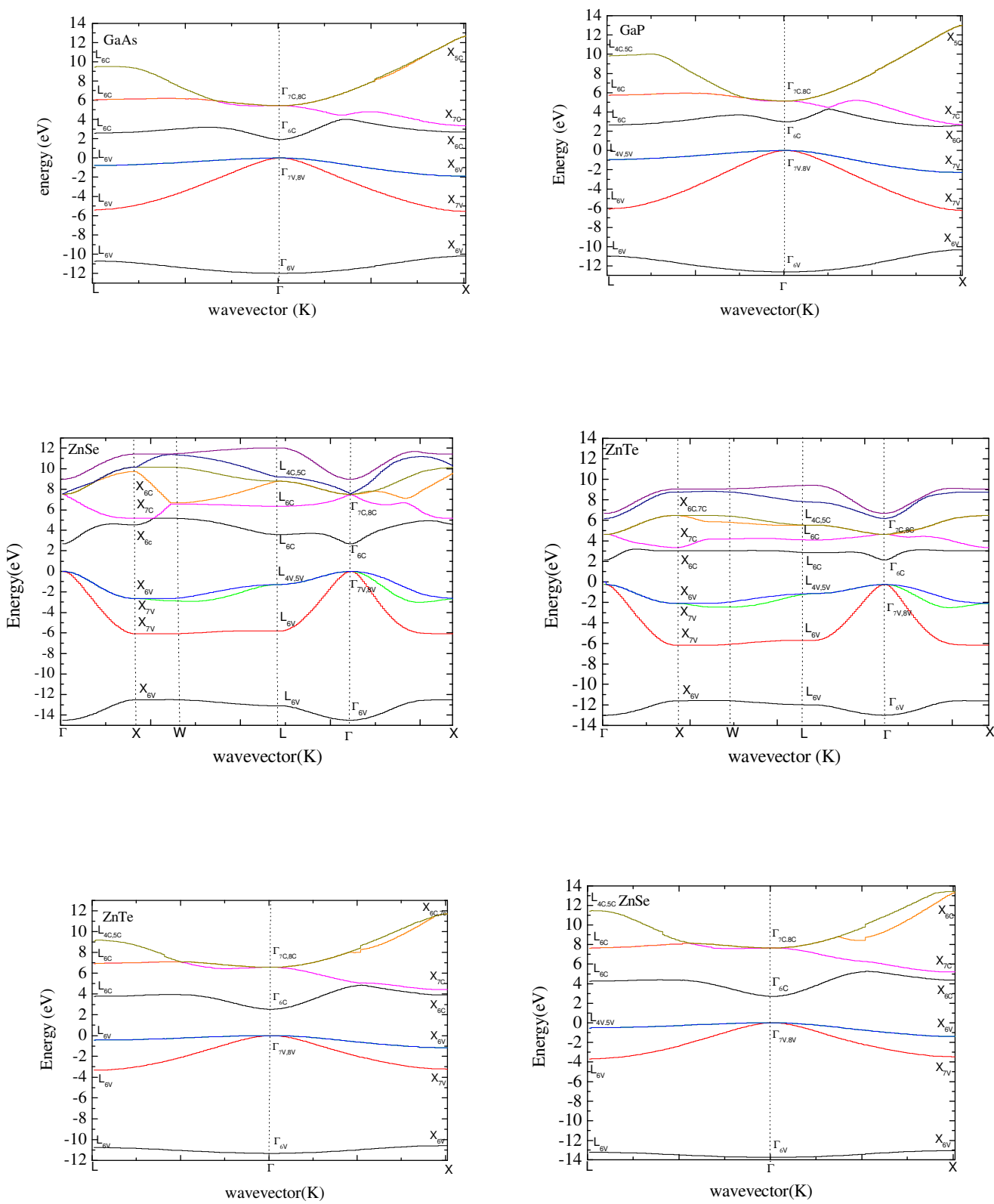

Figure.3 The band structures along the main symmetry directions of $\mathrm{Si}, \mathrm{Ge}, \mathrm{GaAs}, \mathrm{GaP}, \mathrm{ZnSe}, \mathrm{ZnTe}$ by the Tight Binding method and the pseudo potential.

If we compare the two graphs of Si and Ge we find that we are dealing with two types of gaps. A direct gap for the Ge (the minimum of the conduction band and the maximum of the valence band are both at any point representing the center of the Brillouin's zone) and an indirect gap for Si (the minimum the conduction band and the maximum of the valence band are located at points $\mathrm{X}, \Gamma$ respectively). We find the same remark for semiconductor III-V. In fact, GaAs has a direct gap and GaP has an indirect band gap. Semiconductors (II-VI), $\mathrm{ZnTe}$ and $\mathrm{ZnSe}$ are characterized by a direct gap [8].

This information plays a very important role in the use of semiconductors in microelectronics industry.

We consider the case of a semiconductor with direct gap which favors phenomena of recombination of carriers by photon emission and thus is the basis of the value of using these semiconductors for optoelectronic applications. And the width of the direct bandgap is the wavelength of the photons involved. It varies depending on the alloy composition and also on temperature.

In the case of an indirect gap semiconductor, this gap leads to complex phenomena. If absorption of a photon, the electron will first climb vertically in the conduction band and then down into energy by emitting phonons, which will strongly affect the electronic properties and electron-phonon interactions lead. Therefore, there are very poor light emitters which are used for their conductive properties at high temperatures. 


\section{Conclusion}

The comparison between results obtained by both methods (the Tight Binding of TB and the method of pseudo potential (PP) allows us to draw the following conclusions: A good agreement exist between both methods at the morphology of the band structures of valence and conduction of all semiconductors studied (good qualitative description). We must note here that the strategy of the two methods is different: the TB method is developed to work in the direct network while the PP method is developed to work in the reciprocal lattice. The results obtained are very satisfactory and encouraging us to address in future studies the behavior through electronic band structure for complex systems such as heterostructures [9].

\section{References}

1. F. Bloch, Physik 52, 555 (1928).

2. Lowdin, J. Chem. Phys. Rev.18, 1365 (1950)

3. W. Harrison, Electronic Structure and the Proprieties of solids (Freeman, San Francisco 1989).

4. W. Harrison, Phys. Rev. B 4, 2508 (1971)

5. A. Merad, These de Magister, Université Abou-Bakr Belkaid-Tlemcen (2000)

6. M. L. Cohen and J.R Chelikowsky, «in Electronic structure and optical properties of semiconductors», Springer series in solid-state sciences Ed; Springer, new-York (1988)

7. Y. Al-Douri, R. Khenata, Z. Chelahi-Chikr and M. Driz, H. Aourag «Effect Of Spin Orbit on the electronic Properties on Zinc-Blend » J.Physics of Applied 94. DOI: 10.1063/1.1607516 (2003)

8. P. Yu, M. Cardona, fundamentals of semiconductors, Third Edition, Edit Springer (2001)

9. D. Binberg, M. Grundman, and N. N.Ledentsov, Quantum Dot Heterostructures (Chichester: Wiley, 1998) 